\title{
La toute-épice en Europe occidentale, un complément
}

The allspices in occidental Europa, a complement

\section{Françoise Aubaile-Sallenave}

\section{(2) OpenEdition}

\section{Journals}

\section{Édition électronique}

URL : http://journals.openedition.org/ethnoecologie/6382

DOI : $10.4000 /$ ethnoecologie.6382

ISSN : 2267-2419

\section{Éditeur}

Laboratoire Eco-anthropologie et Ethnobiologie

\section{Référence électronique}

Françoise Aubaile-Sallenave, "La toute-épice en Europe occidentale, un complément », Revue d'ethnoécologie [En ligne], 18| 2020, mis en ligne le 28 décembre 2020, consulté le 29 janvier 2021. URL : http://journals.openedition.org/ethnoecologie/6382 ; DOI : https://doi.org/10.4000/ ethnoecologie.6382

Ce document a été généré automatiquement le 29 janvier 2021.

\section{(c) (i) (9)}

Revue d'ethnoécologie est mis à disposition selon les termes de la licence Creative Commons Attribution - Pas d'Utilisation Commerciale - Pas de Modification 4.0 International. 


\title{
La toute-épice en Europe occidentale, un complément
}

The allspices in occidental Europa, a complement

\author{
Françoise Aubaile-Sallenave
}

$1 \quad$ Pimenta dioica (L.) Merr. et P. racemosa (Miller) J. Moore, des Myrtacées, sont deux arbres fort odorants dans toutes leurs parties : ils fournissent la bay-oil des Anglais ${ }^{1}$. En France, on les nomme aussi «quatre-épices $»^{2}$ pour leurs fruits dont la forme évoque le poivre, et l'arôme rappellerait à la fois le clou de girofle, la muscade et la cannelle. C'est sans doute, comme le rapportent Fluckiger et Hanbury (1878, I : 509), une de ces graines que Garrett, droguiste à Londres, donna en 1601 au botaniste Clusius ${ }^{3}$ qui la décrit et la dessine dans son Liber exoticorum. C'est la première référence certaine de cette épice dont la précision du dessin des feuilles à l'apex arrondi, montre que c'est le fruit de $P$. racemosa. 
Figure 1 : Carolus Clusius (1526-1609), présente Pimenta racemosa, à feuilles arrondies

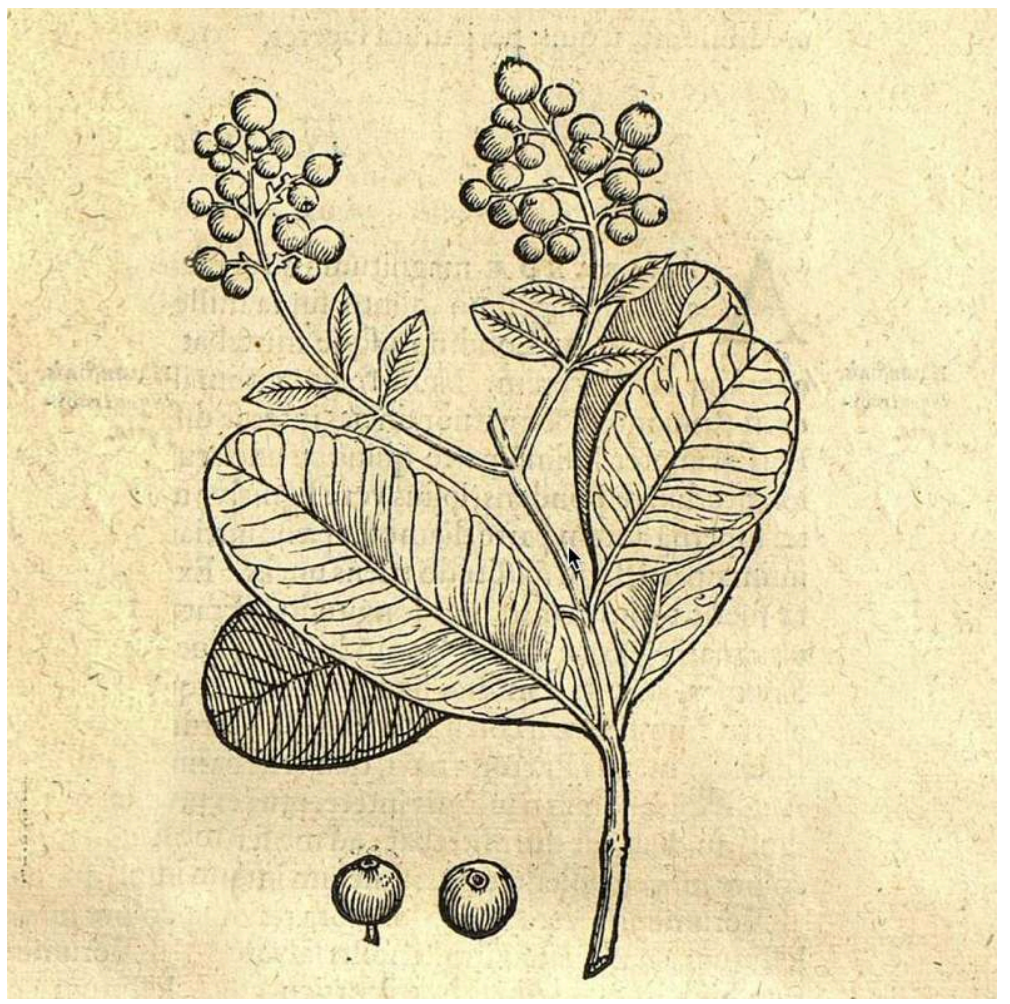

Extr. de : Appendix cultori plantarum exoticarum necessaria, 1630

2 Aujourd'hui, le « bois d'Inde » commercial désigne dans la plupart des cas Pimenta dioica (Bourgeois 1995 : 147). C'est aussi chez les Anglais les all-spice tree, jamaïca-pepper tree, bay-berry tree.

Ce fruit a été nommé « poivre » de la Jamaïque, parce que cette petite graine noire a une odeur piquante. 
Figure 2 : Pimenta dioica (Myristicaceae), cultivé (agriculture biologique)

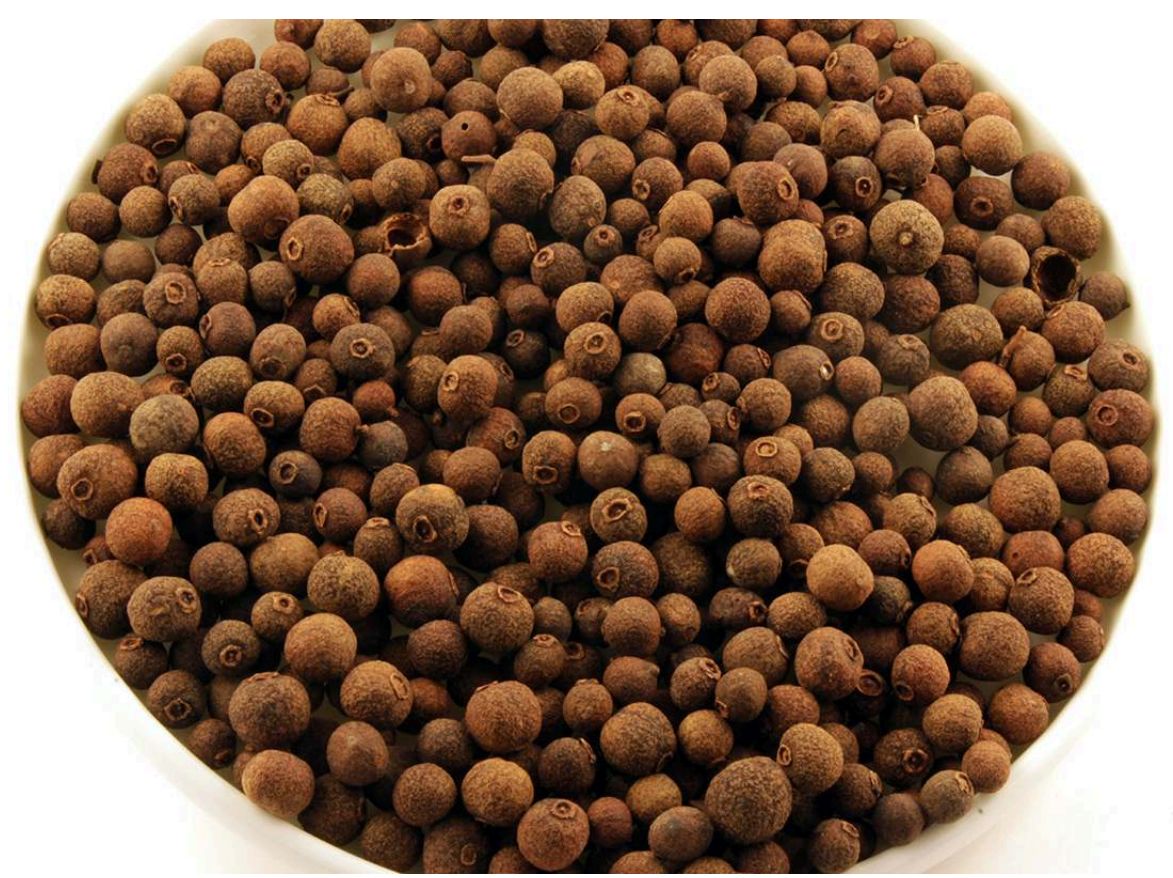

Origine : Coopérative Tosepan Titataniske, Cuetzalan, Puebla, Mexique 22/09/2017 Collecteurs Serge Bahuchet et Pauline Rameau - n ETB-MX-SBPR-2017-85

Cliché S. Bahuchet

On cueille les fruits encore verts mais arrivés à leur plus grand développement, ils ont alors la taille d'un petit pois. Ces fruits distillés donnent une essence dont l'eugénol constitue le principal élément (Baillon 1884). En 1655, les Anglais s'emparent de la Jamaïque et exploitent les nombreux Pimenta dioica qui s'y trouvaient, devenant ainsi les principaux, sinon les seuls importateurs de la graine en Europe. La consommation du Piment de la Jamaïque a été énorme. Pendant l'année 1804-1805, la quantité expédiée des Indes occidentales anglaises a été de 2257000 livres : l'épice du commerce avec l'Europe est alors entièrement fournie par la Jamaïque. Les plantations y sont désignées sous le nom de Pimenta walk, allée de Pimenta, ce sont des terrains naturellement boisés couverts de ces arbres qui n'exigent presque aucune attention (Fluckiger et Hanbury 1878 : I, 509).

5 Les propriétés aromatiques de cette graine sont utilisées à la fois en cuisine, dans certaines liqueurs et en cosmétique.

6 L'Angleterre fut donc longtemps le plus important fournisseur de cette graine à l'Europe. Cette épice, à la saveur brulante et bien corsée, entre dans la cuisine des viandes et des charcuteries, chez les Anglo-Saxons, les Allemands et les Nordiques. Dans ces pays, on l'apprécie également dans les glaces à la vanille, les plum-cakes et autres pâtisseries.

7 Aux États-Unis c'est surtout en cosmétique qu'elle est utilisée ; en effet, les premiers ouvrages de cuisine l'utilisent très peu; The First american Cookbook d'Amelia Simmons (1796) (le premier ouvrage qui ne se base pas sur des ouvrages anglais, à la différence de tous les autres de ce temps), utilise « pepper, black pepper » dans toutes les recettes, " nutmeg, mace, cloves » très souvent, quelquefois « cayenne », mais seulement deux fois « allspice» dans les recettes ci-dessous : 
- page 28 : Pumpkin : one quart of milk, 1 pint pumpkin, 4 eggs, molasses, allspice and ginger in a crust, bake 1 hour.

- page 36 : A cheap seed cake : Rub one pound sugar, half an ounce allspice into four quarters flour, into which pour one pound butter, melted in one pint milk, nine eggs, one gill emptins (carroway seed and currants, or raisins if you please) make into two loaves, bake one and half hour.

Dans le second ouvrage, un peu plus tardif, de Mary Randolf, The Virgina Housewife, 1824, allspice est cité dans deux recettes, l'une de viande de chasse, l'autre d'une conserve :

- page 31: To make hunter's beef, longue recette dont je ne cite que les épices; « 3 ounces saltpetre, one ounce of cloves, half an ounce of alspice (sic), a large nutmeg and a quart of salt » (broyer très fin pour en frotter la viande).

- page 163: Tomato soy. C'est une sorte de sauce tomate qui sera conservée en bouteille ; les tomates macèrent 3 jours, puis sont bouillies un jour au terme duquel on les passe et on y ajoute " one ounce cloves, quarter of a pound of allspice, quarter of a pound of whole black pepper, and a small wine glass of Cayenne». Enfin, on les fait bouillir lentement pendant un jour entier, on les laisse refroidir une nuit puis on les met en bouteilles bien bouchées.

9 En France, au XVIII ${ }^{\mathrm{e}}$ siècle cette épice est donc fournie par les Anglais. Valmont de Bomare, dans son Dictionnaire d'Histoire Naturelle, signale qu'on la nomme " piment des anglais » $(1768$, t. $4: 616)$ et il donne un long article à « poivre de la Jamaïque ou piment des anglais » (1768, t. $5: 127-8)$, « une baie que l'on apporte depuis près de deux siècles de l'île de la Jamaïque et dont les Anglais font un très grand usage dans leurs sauces sous les noms de Toutes épices, Poivre de Thèves, Amomi, Piment à couronnes, Coques d'Inde aromatiques, Tête de clou ... Cet arbre est une espèce de myrte à feuilles de Laurier ». Le texte qu'il donne est traduit de l'anglais, car cette épice transite par l'Angleterre, probablement jusqu'au moins au début du xix siècle.

10 En France, c'est au xix siècle, que la toute-épice entre dans l'aromatisation de liqueurs complexes comme la Chartreuse ${ }^{4}$ et la Bénédictine ${ }^{5}$, pour les plus anciennes, toutes deux ayant à l'origine un rôle médical par les simples qu'elles contiennent.

11 Pimenta racemosa peut devenir, par contre, un grand arbre de 10 à $15 \mathrm{~m}$ de haut; il croît dans les Antilles et sur la côte du Vénézuela, mais il est d'abord surtout exploité à Saint-Domingue, puis dans toutes les Caraïbes (Bourgeois 1995). On en distille les feuilles pour obtenir une huile dont les principaux constituants sont l'eugénol et la vanilline $^{6}$. Le rendement est de 2 à $2,5 \%$ des feuilles sèches; à Saint-Domingue, la production de P. racemosa avoisine 55 tonnes annuelles. Les États-Unis et l'Europe les utilisent principalement dans divers produits cosmétiques, lotions après rasage, eau de Cologne, eaux de toilette, que les Anglo-saxons appellent bay-rum. Cet arbre est le bayrum tree des Anglais. Il est aujourd'hui planté dans toutes les régions tropicales (Baillon 1884, Boullard 1995, Le Florentin 1927, Schwob 1986).

Il est intéressant de noter que ces deux Pimenta sont aujourd'hui essentiellement exploités pour leurs graines et feuilles aromatiques, bien qu'ils aient été d'abord remarqués pour l'odeur parfumée de l'arbre en entier, tronc, écorce, feuilles et puis fruits renfermant les graines. Mais il y a une erreur poursuivie au moins jusqu'en 1694-95 avec les ouvrages de Pomet, épicier français qui n'a jamais vu l'arbre sur pied.

13 En effet, les épiciers dont Pomet considéraient alors que l'arbre donnait trois produits : - le bois de teinture, - les feuilles, - les fruits et les graines parfumés ; on l'a donc aussi appelé Bois de la Jamaïque et Bois d'Inde. 
14 Cet arbre a d'abord été nommé Bois de Campêche, qui s'est révélé être un bois de teinture violet, pourpre, noir, etc., très apprécié aux XVII ${ }^{\mathrm{e}}$ et $\mathrm{XVIII}^{\mathrm{e}}$ siècle pour ces propriétés. C'est en fait Haematoxylon campechianum L., une Fabacée, originaire de la baie de Campêche, du Honduras, et autres régions d'Amérique centrale. Ainsi, en même temps, les graines de ces Pimenta, arbres qui vivaient dans les mêmes zones qu'Haematoxylon campechianum L., ont été également appréciées, surtout par les Anglais, comme épice d'une odeur très proche de celle du girofle.

15 Le texte de Pomet (1695: 121-122) éclaire le nom de Quatre épices que l'on a ensuite donné aux graines en France.

«Ce fruit est connu depuis très peu de temps, nous n'en faisons aucun commerce, mais depuis un an environ, que nos armateurs de St Malo [corsaires, devrait-il dire!] en ont pris une assez bonne quantité sur les Anglois, nous avons peu d'épiciers qui ne connaissent ce fruit sous le nom de Graine de Gerofle, à cause que son goût retire assez à celuy du gerofle, et duquel on commence à se servir pour mettre dans les quatre Epices, et il est vray que ce fruit battu et mis dans quelques sauces, a le même goût que si l'on avait mis du gerofle, de la muscade et de la canelle et avec toutes ces qualités, peu de gens s'en accommodent, soit parce que ce fruit n'est pas encore bien connu, ou que son goût ne plaise pas, ce qui est bien contraire des Anglois qui en usent de très grandes quantités, aussi bien que les Sauvages, qui s'en servent dans leur chocolat ... les Anglois appellent ce fruit Poivre de la Jamaïque et les Hollandois Amomi et nous, fruit du Bois d'Inde et le vulgaire. quoique mal à propos, Graine de Gerofle».

Plus loin au chapitre III des épices fines, Pomet explique ce que sont ces quatre Epices, " pour obvier aux abus qui se glissent dans la composition de ce mélange »:

« Il y a icy à remarquer que la plupart de ceux qui font les quatre Epices emploient au lieu de poivre, de la pousse de poivre; au lieu de girofle, du poivre de la Jamaïque, ou du capelet (?); au lieu de muscade, du costus blanc ou bien d'une certaine écorce dont je n'ai pu savoir ce que c'est sinon qu'elle vient des îles... Je crois que c'est l'écorce de quelques espèces de Sassafras. ...» (Pomet 1695 : 195).

17 On emploie évidemment ce qui est le plus économique, ce qu'était, à ce moment-là, ce poivre de la Jamaïque, Pimenta dioica dont les Anglais, à cette époque, approvisionnaient largement l'Europe et dont Pomet donne l'image intitulée Bois d'Inde, avec ses feuilles à l'apex pointu. 
Figure 3 : Pierre Pomet (1658-1699), présente Pimenta dioica, de la Jamaïque, arbre à feuilles pointues

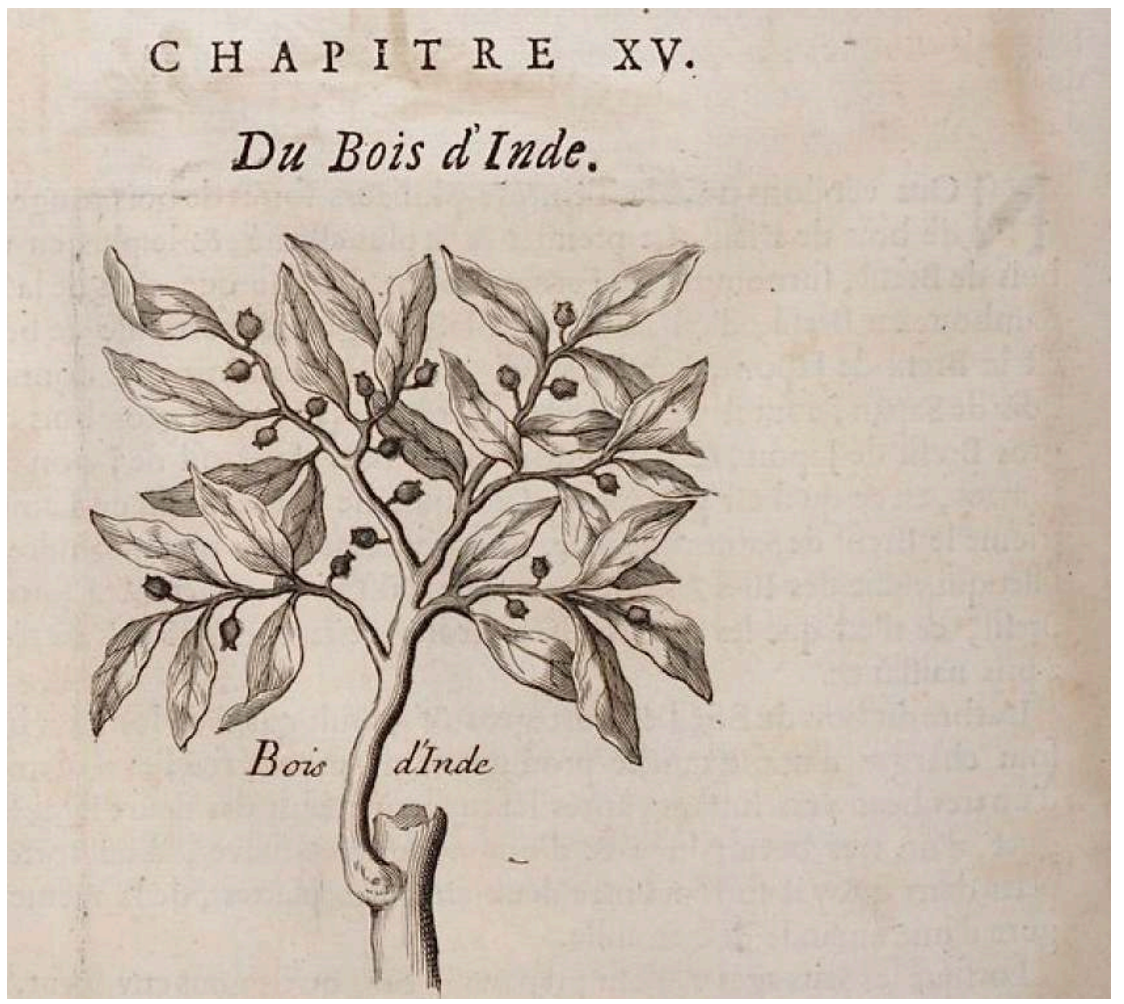

Extr. de : Histoire générale des Drogues 1694 : 120

À la suite de cette mise en garde, Pomet fait un sous chapitre intitulé Le poivre de Thévet ${ }^{7}$, nom que l'on retrouve de temps en temps dans les textes de l'époque. Il décrit le :

« petit fruit rond, de la grosseur du Poivre blanc, d'une couleur rougeâtre, à un des bouts il y a comme une petite couronne... Il est rare et très peu connu ; il est d'un gout aromatique et fort agréable... Les Hollandois ont aussi donné le nom d'Amomi au Poivre cy-dessus, tant à cause de la ressemblance qu'il a avec le Poivre de la Jamaïque, que parce qu'il a presque son même gout. Et à cause qu'il est comme rond et qu'il a le gout du girofle, ... et l'on s'en sert aux mêmes usages, c'est à dire comme du girofle ordinaire. » (Pomet 1695 : 195).

Ce qui nous permet de dire que c'est une Myrtacée et probablement une autre espèce de Pimenta ou une variété de Pimenta dioica.

Planchon et Collins (1895), deux siècles plus tard, distinguent nettement le Piment de la Jamaïque, Pimenta dioica, dont la graine a les quatre lobes séchés du calice à son sommet qui forment une couronne de Amomis acris Berg. et A. pimenta Berg. qui donnent également le Piment couronné, dit encore Poivre de Thévet, dont les fruits, moins aromatiques et un peu plus gros que le Piment de la Jamaïque, sont couronnés par les cinq dents du calice (Planchon \& Collins 1895, II : 335).

21 Et pourtant, les erreurs d'identification de ces graines des Pimenta se retrouvent tardivement encore autant dans le Dictionnaire $d u$ Grand Larousse de Pierre Larousse, 1865-1876 : "La toute-épice. Nom vulgaire de la nigelle cultivée et du myrte-piment » définition reprise exactement en 1900 par le Nouveau Larousse illustré, 7 volumes, sous la direction de Claude Augé. 

(Nigella sativa L. des Renonculacées), qui n'a pas du tout l'arôme ni l'acuité des Pimenta : Pourquoi? on peut inférer qu'en France, on utilise bien peu ces graines de Pimenta en cuisine et donc les Français ne les connaissent pas, bien qu'elles entrent, sans qu'on s'en rendent compte, dans les liqueurs très complexes que sont la Chartreuse et la Bénédictine ainsi que dans les eaux de toilette également très complexes, comme nous l'avons vu plus haut. Par contre, en second lieu, ces deux encyclopédies l'expliquent aussi par le myrte-piment ou Myrtus pimenta, qui est la juste identification de Linné en 1753, signature d'identification remise en question par le jeu du premier descripteur, pour Pimenta dioica, c'est E.D. Merrill (1876-1956), et pour P. racemosa, c'est J.W. Moore (1901-).

italiennes, ces graines à l'arôme si riche en variété ont été très tôt diffusées dans toutes les régions chaudes du monde, autant dans les jardins des particuliers que dans les exploitations économiques, et entrent dans les cuisines tropicales, curries indiens ou colombos antillais. Dans les Caraïbes, elles ont aussi un rôle médicinal (Bourgeois 1995).

\section{BIBLIOGRAPHIE}

Aubaile-Sallenave F. 2002 - Histoire naturelle et culturelle des plantes aromatiques. Paris, Ibis Presse.

Augé C. Ed. 1900 - Le Nouveau Larousse illustré. 7 volumes. Paris, Larousse.

Baillon H. 1884 - Traité de Botanique médicale phanérogamique. Paris, Hachette.

Boullard B. 1995 - La nature des arômes et parfums. Paris, Estem.

Bourgeois P. 1995 - Une plante aromatique de la caraïbe : le bois d'Inde. Journal d'Agriculture Traditionnelle et de Botanique Appliquée 37 (1):139-149.

Clusius Carolus1630 - Appendix cultori plantarum exoticarum necessaria. In : Horstius J. \& Horstius G. Herbarium Horstianum ; seu De selectis plantis et radicibus libri duo. Marburg, Caspar Chemlinus 385-399.

Flückiger F.A. \& Hanbury D. 1878 - Histoire des drogues d'origine végétale. Lanessan trad. du texte anglais Pharmacographia, Paris, Octave Doin, 2 vol.

Lantaz J.-P. 1991 - Bénédictine d'un alambic à cinq continents. Luneray, Bertout.

Larousse P. 1865-1876 - Le Grand Larousse Universel du XIXe siècle. Paris, Larousse.

Le Florentin R. 1927 - Les Parfums. Paris, Desforges, Girardot.

Marseille J. (Ed.) 2002 - Dictionnaire de la Provence et de la Côte d'Azur. Paris, Larousse.

Planchon G. \& Collin E. 1895 - Les drogues simples d'origine végétale. Paris, Gustave Doin.

Pomet 1694 - Histoire générale des drogues... Paris, chez l'auteur.

Pomet 1695 - Le marchand sincère ou traité général des drogues simples et composées. Paris, chez l'auteur.

Revue d'ethnoécologie, 18 | 2020 
Randolf M. 1824 - The Virginia housewife or Methodological cook. New York, Hurst and Company.

Schwob R. 1986 - Essentiel oils and oleoresins: a study of selected produces and major markets. Geneva, International trade Centre Unctad/gatt.

Simmonds A. 1796 - American cooke of the art of dressing viands, fish, poultry and vegetables and the best mode of making pastes, puffs, pie, tarts, puddings, custards and preserves, and all kinds of cakes, from the imperial plumb to plain cake, adapted to this country and all grades of life. Hartford, Hudson \& Goodwyn.

[Société de gens de lettres] 1764 - Dictionnaire domestique portatif. Paris, chez Vincent, 3 vol.

Valmont de Bomare 1768 - Dictionnaire d'Histoire Naturelle. Paris, 6 vol.

\section{NOTES}

1. Je reprends la notice intitulée « Toute-épice » dans Aubaile $2002: 142$.

2. Quatre épice, Toutes épices, allspices des Anglais, sont les noms donnés très tôt à cette épice qui pourtant exhale un parfum très marqué de girofle (Syzygium aromaticum, une Myrtacée, de même famille que Pimenta) avec des notes beaucoup moins sensibles de cannelle (Cinnamomum, une Lauracée) et de poivre, une Pipéracée, par son arôme assez agressif.

3. Clusius, nom latin de Charles de Lécluse, né à Arras en 1526, est étudiant à Montpellier. Après plusieurs fonctions en Europe, il est nommé professeur de botanique à Leyden en 1589 et le reste jusqu'à sa mort en 1609 ; il y crée le jardin botanique dont une partie subsiste encore de nos jours.

4. La Chartreuse est la plus ancienne de ces deux liqueurs. Elle est aujourd'hui fabriquée à la distillerie d'Aiguenoire à Entre-deux-Guiers en Isère, en plein cœur du massif de la Chartreuse, sous la supervision des moines de la Grande-Chartreuse. Liqueur à très haut degré d'alcool $\left(55^{\circ}\right.$ pour la verte), sa vente est la principale ressource financière des Chartreux. La recette complète de cet élixir reste secrète. (Marseille $2002: 278$ ).

5. La Bénédictine est une boisson alcoolisée digestive, fabriquée à Fécamp en Normandie. La société de production appartient aujourd'hui au groupe Bacardi-Martini France. Son histoire n'est pas très ancienne et pourrait être liée à la dispersion de l'ordre des Bénédictins de Fécamp lors de la Révolution française, et d'un manuscrit retrouvé en 1863 où serait notée une recette de cette liqueur, mise au point par Alexandre le Grand qui crée un nouvel «élixir de santé » qu'il baptise Bénédictine. (Lantaz 1991 : 18).

6. Bourgeois, dans son étude récente, 1995, détaille les propriétés aromatiques variées des feuilles, des fruits et des petits bois.

7. Le Dictionnaire domestique portatif le cite simplement comme poivre de la Jamaïque ou poivre de Thevet (sic). Ce court article descriptif se termine par "il est un des meilleurs aromates». (Société des gens de lettres $2: 402$ ).

\section{RÉSUMÉS}

Ce court article est un complément du précédent (Machuca et al. ce numéro), faisant l'historique de l'arrivée des graines de Pimenta en Europe, du rôle primordial des Anglais, de sa 
méconnaissance en cuisine française et américaine mais de ses usages dans certaines liqueurs et en cosmétique.

This short paper complements information about historical introduction of the seeds of Pimenta in Europa, the important paper of the English, the nearly ignorance of the seeds in French and American cooking, but their uses in some liqueurs and in cosmetic.

INDEX

Mots-clés : Pimenta, histoire, cuisine, liqueur, cosmétique

Keywords : Pimenta, history, cooking, liqueur, cosmetic

\section{AUTEUR}

\section{FRANÇOISE AUBAILE-SALLENAVE}

UMR 7206, Musée de l'homme 